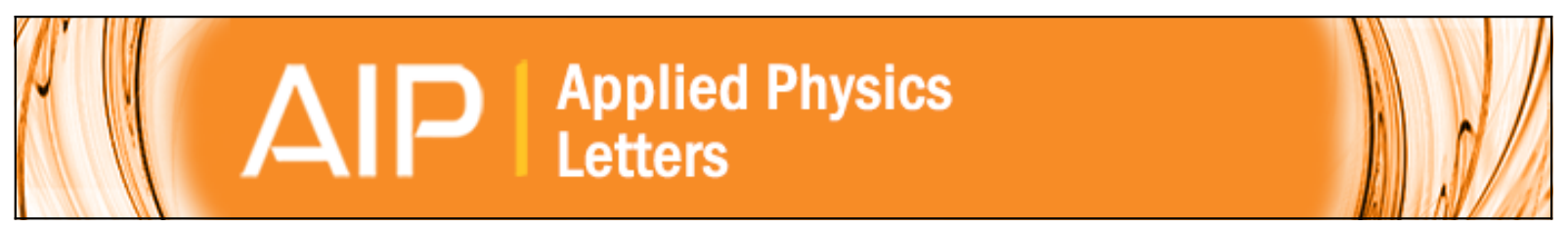

\title{
Stranski-Krastanov InN/InGaN quantum dots grown directly on $\mathrm{Si}(111)$
}

Paul E. D. Soto Rodriguez, Pavel Aseev, Victor J. Gómez, Praveen Kumar, Naveed UI Hassan Alvi, Enrique Calleja, José M. Mánuel, Francisco M. Morales, Juan J. Jiménez, Rafael García, Alexander Senichev, Christoph Lienau, and Richard Nötzel

Citation: Applied Physics Letters 106, 023105 (2015); doi: 10.1063/1.4905662

View online: http://dx.doi.org/10.1063/1.4905662

View Table of Contents: http://scitation.aip.org/content/aip/journal/apl/106/2?ver=pdfcov

Published by the AIP Publishing

\section{Articles you may be interested in}

Micro-Raman investigations of InN-GaN core-shell nanowires on Si (111) substrate

AIP Advances 3, 062114 (2013); 10.1063/1.4811365

Spontaneous formation of InGaN nanowall network directly on Si

Appl. Phys. Lett. 102, 173105 (2013); 10.1063/1.4803017

Thermal stability of the deep ultraviolet emission from AIGaN/AIN Stranski-Krastanov quantum dots Appl. Phys. Lett. 101, 241914 (2012); 10.1063/1.4770075

Internal quantum efficiency of III-nitride quantum dot superlattices grown by plasma-assisted molecular-beam epitaxy

J. Appl. Phys. 109, 103501 (2011); 10.1063/1.3590151

Investigation of AIN films grown by molecular beam epitaxy on vicinal $\mathrm{Si}(111)$ as templates for GaN quantum dots

Appl. Phys. Lett. 89, 231903 (2006); 10.1063/1.2399940

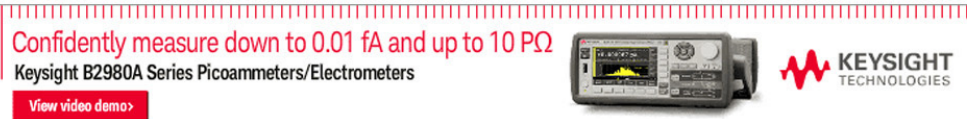




\title{
Stranski-Krastanov InN/InGaN quantum dots grown directly on Si(111)
}

\author{
Paul E. D. Soto Rodriguez, ${ }^{1, a)}$ Pavel Aseev, ${ }^{1}$ Victor J. Gómez, ${ }^{1}$ Praveen Kumar, ${ }^{1}$ \\ Naveed UI Hassan Alvi, ${ }^{1}$ Enrique Calleja, ${ }^{1}$ José M. Mánuel, ${ }^{2}$ Francisco M. Morales, ${ }^{2,3}$ \\ Juan J. Jiménez, ${ }^{2}$ Rafael García, ${ }^{2}$ Alexander Senichev, ${ }^{4}$ Christoph Lienau, ${ }^{5}$ \\ and Richard Nötzel ${ }^{6, b)}$ \\ ${ }^{1}$ Instituto de Sistemas Optoelectrónicos y Microtecnología (ISOM), Universidad Politécnica de Madrid, \\ Ciudad Universitaria s/n, 28040 Madrid, Spain \\ ${ }^{2}$ Dep. Ciencia de los Materiales e IM y QI., F. Ciencias, Universidad de Cádiz, 11510-Puerto Real, Cádiz, \\ Spain \\ ${ }^{3}$ IMEYMAT: Institute of Research on Electron Microscopy and Materials of the University of Cádiz, Cádiz, \\ Spain \\ ${ }^{4}$ Max Planck Institute of Microstructure Physics, Weinberg 2, Halle 06120, Germany \\ ${ }^{5}$ Institut für Physik, Carl von Ossietzky Universität Oldenburg, 26129 Oldenburg, Germany \\ ${ }^{6}$ L-NESS and Dipartimento di Scienza dei Materiali, Universitá di Milano-Bicocca, Via Cozzi 53, 20125 \\ Milano, Italy
}

(Received 25 November 2014; accepted 26 December 2014; published online 12 January 2015)

\begin{abstract}
The authors discuss and demonstrate the growth of InN surface quantum dots on a high-In-content $\mathrm{In}_{0.73} \mathrm{Ga}_{0.27} \mathrm{~N}$ layer, directly on a $\mathrm{Si}(111)$ substrate by plasma-assisted molecular beam epitaxy. Atomic force microscopy and transmission electron microscopy reveal uniformly distributed quantum dots with diameters of $10-40 \mathrm{~nm}$, heights of $2-4 \mathrm{~nm}$, and a relatively low density of $\sim 7 \times 10^{9} \mathrm{~cm}^{-2}$. A thin InN wetting layer below the quantum dots proves the Stranski-Krastanov growth mode. Nearfield scanning optical microscopy shows distinct and spatially well localized near-infrared emission from single surface quantum dots. This holds promise for future telecommunication and sensing devices. (C) 2015 AIP Publishing LLC. [http://dx.doi.org/10.1063/1.4905662]
\end{abstract}

Research on semiconductor nanomaterials has led to a vast variety of applications, including renewable energy, bio-sensing, and nano-photonics. In the search for the best material, InGaN combines the most superior properties such as the widest bandgap tunability, high near band edge absorption, high carrier mobility, surface electron accumulation, chemical stability, and superior radiation hardness. ${ }^{1}$ High flexibility in performance design and functionality is offered by the combination with quantum dots (QDs) acting as artificial atoms with size dependent quantized electronic properties. Self-assembled GaN QDs have been intensively investigated for blue light emitters. ${ }^{2}$ In the pursuit of high performance green light emitting diodes, InGaN QDs have been grown on both $c$ - and $m$-plane $\mathrm{GaN}^{3,4}$ and by selective area epitaxy applying diblock copolymer lithography. ${ }^{5}$ InGaN QDs on GaN have been obtained by both molecular beam epitaxy (MBE) and metal organic vapor phase epitaxy (MOVPE) by either direct island formation (Volmer-Weber, VW growth mode) or wetting layer followed by island formation (Stranski-Krastanov, SK growth mode). ${ }^{6-9}$

Near-infrared photoluminescence (PL) emission was reported for InN QDs on GaN with size dependent peak energy, blue shifting from 0.78 to $1.07 \mathrm{eV}$ when the QD height was reduced from 32.4 to $6.5 \mathrm{~nm} .{ }^{10}$ We have recently started the growth of InN QDs on high-In-content InGaN layers by plasma-assisted (PA) MBE and found excellent optical performance optimized for intermediate band solar cells and demonstrated applications in the fields of bio-sensors and ion-selective electrodes. ${ }^{11-14}$ As for most of the previous

\footnotetext{
a)p.soto@isom.upm.es

b)noetzel500@gmail.com
}

studies, these QD structures were grown on GaN/sapphire templates. This limits device design flexibility due to the insulating behavior. Hence, the direct growth on $\mathrm{Si}$ substrates is the ultimate goal, allowing for device designs such as InGaN/Si tandem solar cells and vertical contact schemes at much lower cost and for the direct integration with existing Si technology. Along this line, we have already reported the growth of thick and uniform high-In-content InGaN layers on Si by either strongly promoting growth selectivity (the separation of low- and high-In-content regions) producing micron-sized atomically flat In-rich regions ${ }^{15}$ or by the suppression of growth selectivity at lower temperature resulting in undulated, chemically uniform InGaN layers ${ }^{16}$ with high optical quality.

Here, we report the growth of InN surface QDs (SQDs) on such a chemically uniform high-In-content $\mathrm{In}_{0.73} \mathrm{Ga}_{0.27} \mathrm{~N}$ layer (the In content is determined by X-ray diffraction), directly on $\mathrm{Si}(111)$ by PA MBE. Atomic force microscopy (AFM) and transmission electron microscopy (TEM) reveal uniformly distributed QDs with diameters ranging from 10 to $40 \mathrm{~nm}$ and a relatively low density of $\sim 7 \times 10^{9}$ QDs cm ${ }^{-2}$. AFM measurements show QD heights of 2-4 nm. Formation of a thin $(0.9 \mathrm{~nm})$ and uniform two-dimensional $\mathrm{InN}$ wetting layer proves the Stranski-Krastanov growth mode. Near-field scanning optical microscopy (NSOM) measurements show spatially uniform emission from the InGaN layer and distinct and spatially well localized emission from single SQDs in the near-infrared. The QDs structural quality is comparable to that of the QDs grown on GaN/sapphire templates.

All growth experiments were performed in a PA MBE [MECA 2000] system equipped with a radio-frequency (RF) active $\mathrm{N}$ plasma source. 1 in. $\mathrm{Si}(111)$ wafers were degassed 

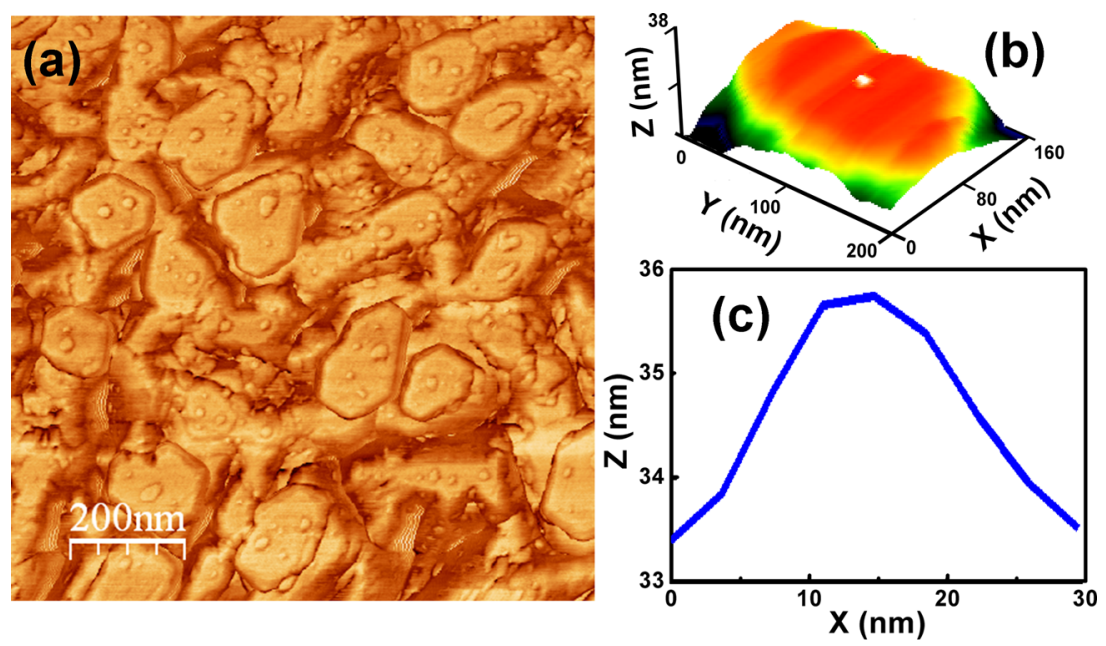

FIG. 1. (a): $1 \times 1 \mu \mathrm{m}^{2}$ AFM image of the InN SQD/ $/ \mathrm{In}_{0.73} \mathrm{Ga}_{0.27} \mathrm{~N}$ layer sample surface. (b) Three-dimensional AFM image with enlarged magnification. (c) AFM line scan across the SQD.

in the introduction chamber at $450{ }^{\circ} \mathrm{C}$ for $4 \mathrm{~h}$ followed by annealing at $850^{\circ} \mathrm{C}$ for $30 \mathrm{~min}$ in the growth chamber for oxide removal. The cleaned $\mathrm{Si}$ surface was then nitrided under active $\mathrm{N}$ flux with molecular $\mathrm{N}_{2}$ flow rate of $0.9 \mathrm{sccm}$ and $\mathrm{RF}$ power of $350 \mathrm{~W}$ at $850{ }^{\circ} \mathrm{C}$ for $5 \mathrm{~min}$. After cooling down to $450{ }^{\circ} \mathrm{C}$, InGaN growth was performed with the $\mathrm{Ga}$, In, and active $\mathrm{N}$ fluxes of $8.1 \times 10^{13}, 4.4 \times 10^{14}$, and $7.2 \times 10^{14}$ atoms $/ \mathrm{cm}^{2} \mathrm{~s}$, respectively. The growth time was $1 \mathrm{~h}$ resulting in a $450 \mathrm{~nm}$ thick and compact InGaN layer. For the InN SQDs growth, the same In and $\mathrm{N}$ fluxes were maintained and no growth interruption was inserted. The growth time for the SQDs was $10 \mathrm{~s}$ ( $\sim 3$ monolayers).

The surface morphology was investigated by AFM in tapping mode in air. For the TEM investigations, the samples were prepared in cross-sectional orientations and thinned to electron transparency by mechanical grinding, polishing, and ion milling at $3 \mathrm{keV}$ with a Gatan low-voltage precision ion polishing system. The high-resolution TEM (HRTEM) results were collected in a JEOL 2010 FEG microscope operated at $200 \mathrm{keV}$. The optical properties were studied using a home built low-temperature NSOM operating at $10 \mathrm{~K}$ inside a high vacuum chamber, described in detail elsewhere. ${ }^{17}$ The sample was excited by a 543-nm He-Ne laser. The laser light was coupled into an uncoated, single mode near-field fiber probe from Nanonics resulting in a laser power in the fiber of $3.9 \mu \mathrm{W}$. The PL from the sample was collected through the same fiber, providing a spatial resolution of about $150 \mathrm{~nm} .^{18}$ The signal was dispersed in an imaging monochromator and recorded with a liquid-nitrogen-cooled InGaAs (OMA V) detector.

Figure 1(a) shows a $1 \times 1 \mu \mathrm{m}^{2}$ AFM image of the InGaN and SQDs surface morphology. The InGaN surface comprises $100-200 \mathrm{~nm}$ wide plateaus together with uniformly distributed InN SQDs. The development of these plateaus, ideally suited for QD formation, points to the transition to the columnar growth regime for higher active $\mathrm{N}$ fluxes, but the present InGaN layer is still compact. A threedimensional AFM image with enlarged magnification of one of the plateaus with a single SQD on top is presented in Fig. 1(b). The corresponding AFM line scan across the InN SQD is depicted in Fig. 1(c). By averaging over line scans recorded on a large number of different individual SQDs, we infer a SQD diameter ranging from 10 to $40 \mathrm{~nm}$ and height of 2-4 nm. The average density of the SQDs, deduced from AFM, is $7 \times 10^{9}$ QDs cm $^{-2}$.

Detailed atomic-scale structural analysis is provided by the cross-sectional HRTEM image of a single SQD, shown in Fig. 2(a). The QD, indicated by the blue line is $3 \mathrm{~nm}$ high and has a base width of $10 \mathrm{~nm}$. The continuous twodimensional $\mathrm{InN}$ wetting layer underneath the QD, indicated by the red lines, is about $0.9 \mathrm{~nm}$ thick. The crystal structure of both the wetting layer and the top portion of the QD is cubic, whereas the crystal structure of the InGaN layer and lower QD portion is hexagonal or, for the QD, contains a
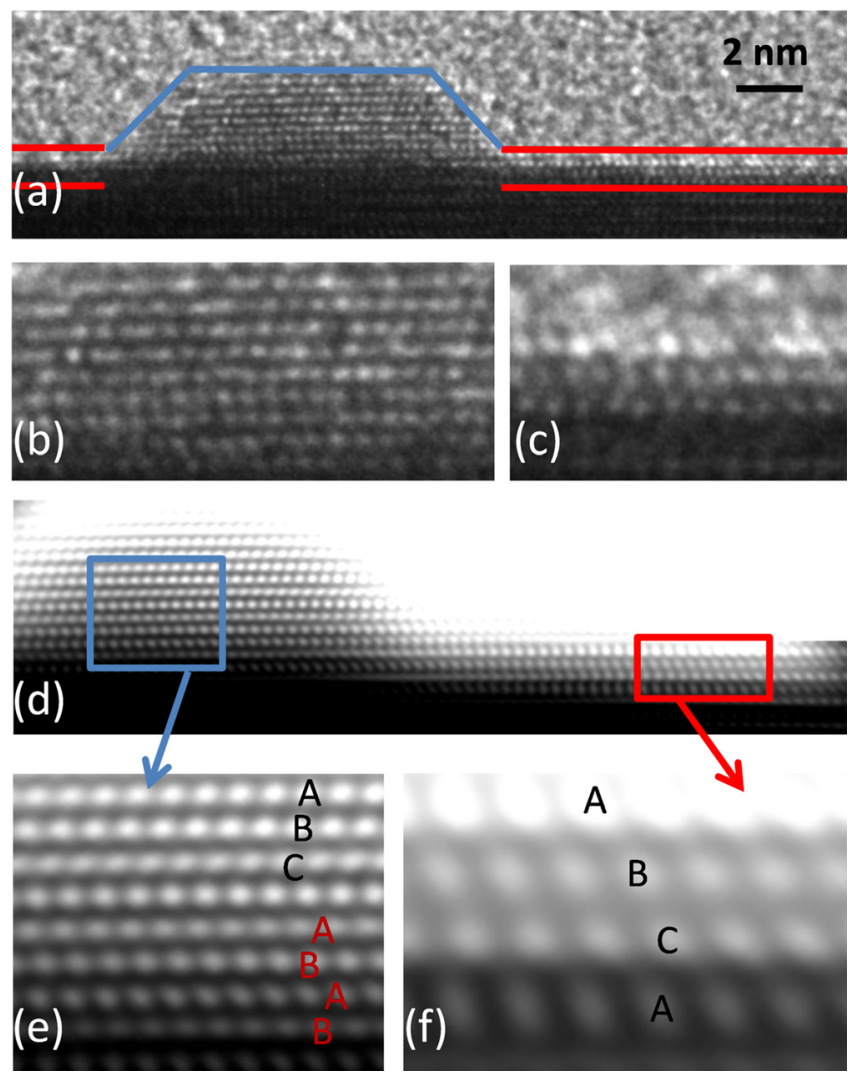

FIG. 2. (a) HRTEM image of an $\mathrm{InN} S Q D / \mathrm{In}_{0.73} \mathrm{Ga}_{0.27} \mathrm{~N}$ layer directly grown on $\mathrm{Si}(111)$ taken along the ${ }^{11-20}$ III-N zone axis, highlighting SQD (blue line), and WL (red line). (b) Zoom of the HRTEM for the QD and (c) for the WL. (d) Filtered image HRTEM. (e) Zoom indicating the cubic ( $\mathrm{ABCABC}$ stack) and hexagonal structure (ABAB stack) for the $\mathrm{QD}$ and (d) the cubic structure (ABCABC stack) for the WL. 

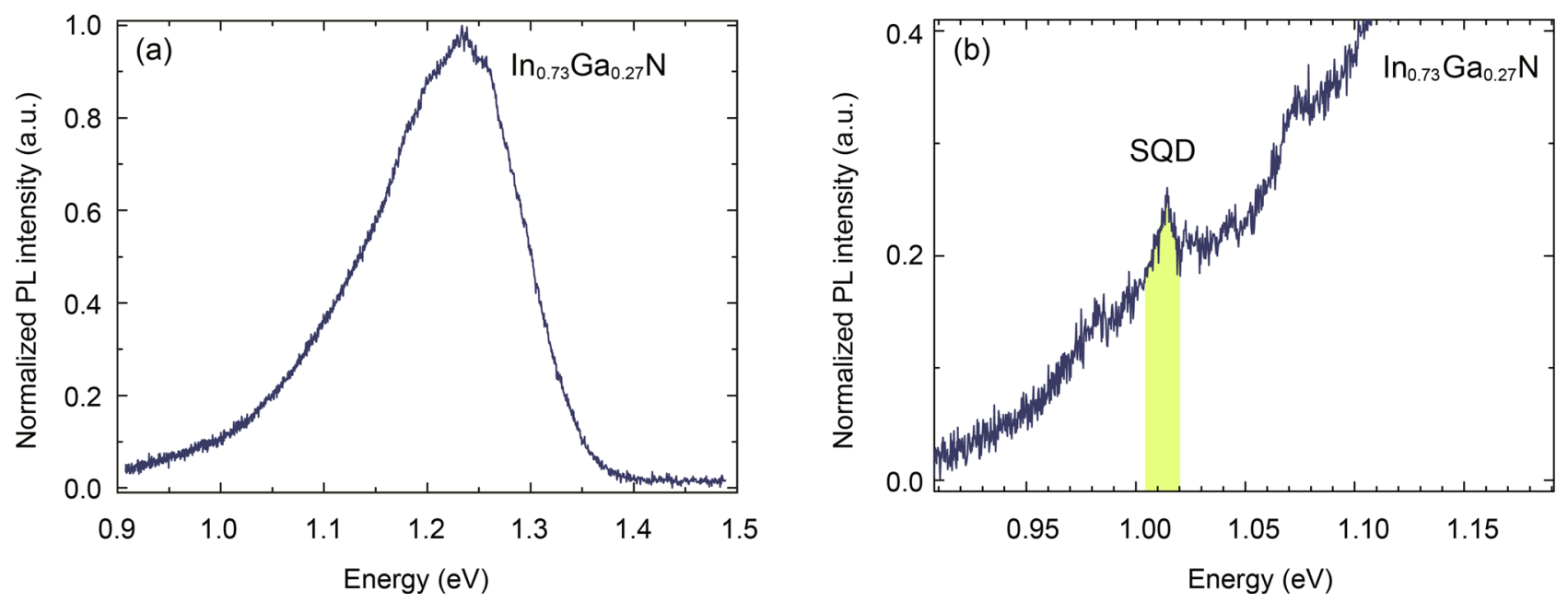

FIG. 3. (a) Near-field PL spectrum acquired at an arbitrarily chosen position of the sample related to the emission from the InGaN layer. (b) Selected near-field PL spectrum showing a distinct emission peak from a single SQD on the low energy tail of the InGaN emission.

mixture of the cubic and hexagonal phases, as can be observed in Figs. 2(b) and 2(c), which are a detail of the QD and the wetting layer, respectively. This implies a change of the atomic stacking sequence $\mathrm{ABAB}$ in the case of an hexagonal crystal, and $\mathrm{ABCABC}$, for a cubic one, when they are aligned along the [112̄0] and [111] zone axis, respectively) upon initiation of the $\mathrm{InN}$ growth, while the successive relaxation by formation of the three-dimensional QD results in the introduction of stacking faults, i.e., a mixing of the initial hexagonal structure of the InGaN layer and the cubic structure of the thin InN layer. To better observe the atomic structure, the image in Fig. 2(a) has been filtered (using a conventional spot mask on the Fast-Fourier transform of the image), which led to Fig. 2(d), and its details, Figs. 2(e) and 2(f); it is possible to easily appreciate the cubic and hexagonal regions forming the QD and wetting layer.

Formation of a wetting layer, i.e., growth in the SK mode is attributed to the medium lattice mismatch for the current materials combination which is very close to that of the InAs/GaAs system, which is the most widely studied SK model system. Lower mismatch easily results in an undulated surface instead of QD nucleation, while larger mismatch favors the VW growth mode. The appearance of a cubic wetting layer at the initiation of $\mathrm{InN}$ growth requires further studies. It might be related to the abrupt increase of the group V to III flux ratio when switching to InN growth (closing of the Ga shutter) ${ }^{19}$ or the high compressive strain in the InN layer. Certainly, in view of applications, this behavior is beneficial due to the absence of piezoelectric fields in cubic structures. ${ }^{20}$

Figure 3(a) shows a near-field overview PL spectrum taken at an arbitrary position. This broad PL spectrum with peak energy of $1.23 \mathrm{eV}$ is due to emission from the $\mathrm{InGaN}$ layer as confirmed by reference spectra of samples without SQDs. At selected positions on the sample in the energy range from 0.95 to $1.10 \mathrm{eV}$, the emergence of narrow PL peaks is observed. These peaks are tentatively attributed to the emission of distinct SQDs. A representative PL spectrum of a SQD is shown in Fig. 3(b) with a peak width of $15 \mathrm{meV}$. These assignments to single SQD emission are directly confirmed by spatial maps of the PL intensity. In Fig. 4(a), a spatial map of the PL intensity related to the InGaN layer is shown, at the energy of $1.19 \mathrm{eV}$ outside of the SQDs energy region, revealing spatially uniform emission intensity in this area. The near-field PL intensity map for the individual SQDs with PL intensity integrated from 1.00 to $1.08 \mathrm{eV}$, is shown in Fig. 4(b). Several well localized emission centers are observed. The density of optically active (detected)
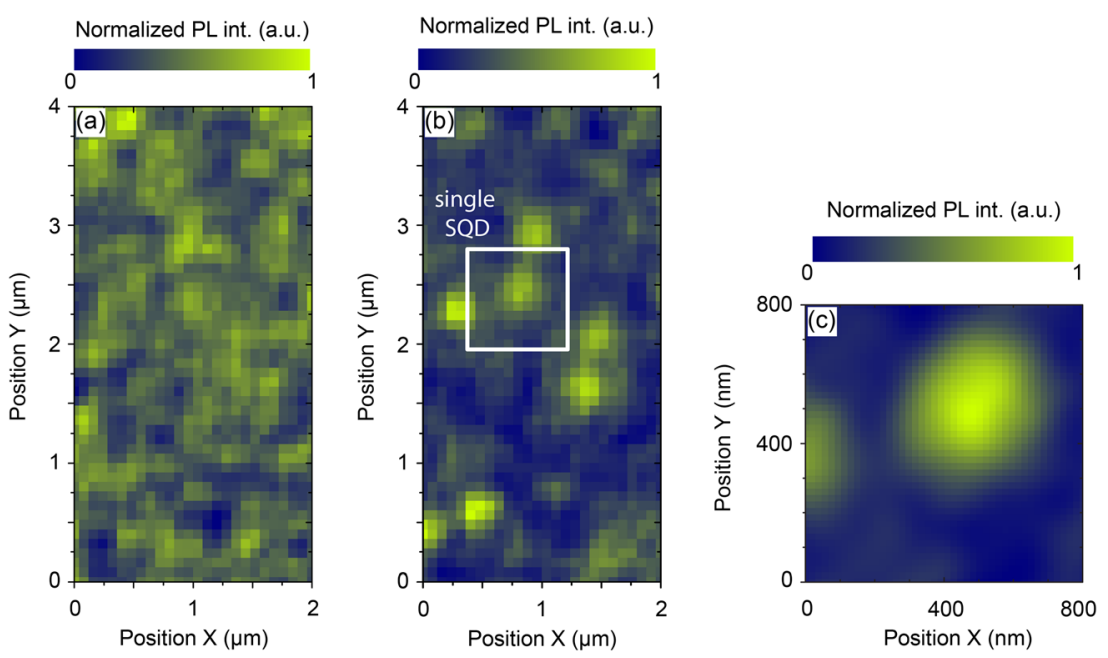

FIG. 4. Near-field map of the PL intensity acquired at $10 \mathrm{~K}$ for (a) the $\mathrm{InGaN}$ layer at energy $1.19 \mathrm{eV}$ outside the energy region of the SQDs, (b) individual SQDs integrated over the energy range of $1.00-1.08 \mathrm{eV}$, and (c) the single SQD with enlarged magnification integrated from 1.005 to $1.020 \mathrm{eV}$. 
SQDs is smaller than the SQD density deduced from AFM, indicating that not all SQDs are optically active. The PL peak presented in Fig. 3(b) stems from the localized emission center in the area indicated by the white square in Fig. 4(b). The corresponding near-field PL intensity map of the single SQD with enlarged magnification is presented in Fig. 4(c) for the PL intensity integrated from 1.005 to $1.020 \mathrm{eV}$ (Fig. 3(b), shadowed area). Evidently, single SQD emission is spatially and spectrally well resolved. The PL peak width for the single SQD of $15 \mathrm{meV}$ is wider than that of the highest purity single QDs being in the order of $\mu \mathrm{eV}$ even for single InGaN QD. ${ }^{21}$ This is typical for SQDs due to the nearby presence of charged surface states. Temporal variations in the charge distribution within these states give rise to fluctuating electric fields in the confined SQDs and thus to a line broadening in continuous wave PL measurements. InN has the highest density of charged surface states among all semiconductors and such line broadening effects are, therefore, particularly pronounced. This is in line with the reported 3$\mathrm{meV}$ peak width for single InAs/GaAs SQDs ${ }^{22}$ and the 9meV peak width for single InGaN SQDs with $20 \%-25 \%$ In content. ${ }^{23}$ Most useful, this sensitivity of the energy states of SQDs to the charge environment with the highest surface state density for InN SQDs is at the heart for highly sensitive sensing applications.

In conclusion, InN SQDs have been grown on high-Incontent $\operatorname{In}_{0.73} \mathrm{Ga}_{0.27} \mathrm{~N}$ layers, directly on $\mathrm{Si}(111)$ by plasmaassisted molecular beam epitaxy. Atomic force microscopy and transmission electron microscopy revealed uniformly distributed QDs with diameters of $10-40 \mathrm{~nm}$, heights of $2-4 \mathrm{~nm}$, and a rather low density of $\sim 7 \times 10^{9} \mathrm{QDs} \mathrm{cm}^{-2}$. The Stranski-Krastanov growth mode was evidenced by the presence of a thin two-dimensional InN wetting layer below the QDs. Near-field scanning optical microscopy showed uniform emission from the InGaN layer and distinct and spatially well localized emission from single SQDs in the nearinfrared spectral range. Such properties have high potential for telecommunication and highly efficient sensing devices.

P.K. acknowledges a Marie Curie Fellowship from the EC. C.L. gratefully acknowledges financial support by the DFG (SPP 1391, Li580/8-1) and by the European Union project CRONOS (Grant No. 280879-2). TEM experiments were carried out in the Electron Microscopy (DME) and
Sample Preparation (LPM) Divisions of the Central Services of Science and Technology of the University of Cádiz (SCCYT-UCA).

${ }^{1}$ J. Wu, J. Appl. Phys. 106, 011101 (2009).

${ }^{2}$ F. Widmann, J. Simon, B. Daudin, G. Feuillet, J. L. Rouvière, N. T. Pelekanos, and G. Fishman, Phys. Rev. B 58, R15989 (1998).

${ }^{3}$ I. K. Park and S. J. Park, Appl. Phys. Express 4, 042102 (2011).

${ }^{4}$ X. Yang, M. Arita, S. Kako, and Y. Arakawa, Phys. Status Solidi C 9, 613 (2012).

${ }^{5}$ G. Liu, H. Zhao, J. Zhang, J. Park, L. Mawst, and N. Tansu, Nanoscale Res. Lett. 6, 342 (2011).

${ }^{6}$ B. Damilano, N. Grandjean, S. Dalmasso, and J. Massies, Appl. Phys. Lett. 75, 3751 (1999).

${ }^{7}$ C. Adelmann, J. Simon, G. Feuillet, N. T. Pelekanos, B. Daudin, and G. Fishman, Appl. Phys. Lett. 76, 1570 (2000).

${ }^{8}$ C. Kruse, S. Figge, and D. Hummel, "Growth of II-VI and III-nitride quantum-dot microcavity systems," in Optics with Semiconductor Nanostructures, edited by F. Jahnke (Woodhead Publishing Limited, Cambridge, 2012), p. 447.

${ }^{9}$ W. Zhao, L. Wang, J. Wang, W. Lv, Z. Hao, and Y. Luo, Phys. Status Solidi A 209, 1096 (2012).

${ }^{10}$ W. C. Ke, C. P. Fu, C. Y. Chen, L. Lee, C. S. Ku, W. C. Chou, W. H. Chang, M. C. Lee, W. K. Chen, W. J. Lin, and Y. C. Cheng, Appl. Phys. Lett. 88, 191913 (2006).

${ }^{11}$ P. E. D. Soto Rodriguez, V. Gómez, P. Kumar, E. Calleja, and R. Nötzel, Appl. Phys. Lett. 102, 131909 (2013).

${ }^{12}$ N. Alvi, P. E. D. Soto Rodriguez, V. J. Gómez, P. Kumar, G. Amin, O. Nur, M. Willander, and R. Nötzel, Appl. Phys. Lett. 101, 153110 (2012).

${ }^{13}$ N. Alvi, P. E. D. Soto Rodriguez, V. J. Gómez, P. Kumar, M. Willander, and R. Nötzel, Appl. Phys. Express 6, 115201 (2013).

${ }^{14}$ N. Alvi, V. J. Gómez, P. E. D. Soto Rodriguez, P. Kumar, S. Zaman, M. Willander, and R. Nötzel, Sensors 13, 13917 (2013).

${ }^{15}$ P. Kumar, P. E. D. Soto Rodriguez, V. J. Gómez, N. H. Alvi, E. Calleja, and R. Nötzel, Appl. Phys. Express 6, 035501 (2013).

${ }^{16}$ P. Aseev, P. E. D. Soto Rodriguez, P. Kumar, V. J. Gómez, N. H. Alvi, J. M. Mánuel, F. M. Morales, J. J. Jiménez, R. García, E. Calleja, and R. Nötzel, Appl. Phys. Express. 6, 115503 (2013).

${ }^{17}$ G. Behme, A. Richter, M. Süptitz, and Ch. Lienau, Rev. Sci. Instrum. 68, 3458 (1997).

${ }^{18}$ F. Intonti, V. Emiliani, C. Lienau, T. Elsaesser, R. Nötzel, and K. Ploog, Phys. Rev. B 63, 075313 (2001).

${ }^{19}$ C. G. Zhang, L. F. Bian, W. D. Chen, and C. C. Hsu, J. Cryst. Growth 299, 268 (2007).

${ }^{20}$ J. Schörmann, D. J. As, and K. Lischka, 2006 MRS Fall Meeting Symposium I - Advances in III-V Nitride semiconductor Materials and Devices (MRS Symp. Proc., 2007), Vol. 955, p. I8.3.

${ }^{21}$ O. Moriwaki, T. Someya, K. Tachibana, S. Ishida, and Y. Arakawa, Appl. Phys. Lett. 76, 2361 (2000).

${ }^{22}$ E. Selçuk, G. J. Hamhuis, and R. Nötzel, AIP Conf. Proc. 1199, 535 (2010).

${ }^{23}$ J. Robinson, J. Rice, K. Lee, J. Na, R. Taylor, D. Hasko, R. Oliver, M. Kappers, C. Humphreys, and G. Briggs, Appl. Phys. Lett. 86, 213103 (2005). 